\title{
Room Temperature Selective Reduction of Nitrobenzene to Azoxybenzene over Magnetically Separable Urchin-like Ni /Graphene Nanocomposites
}

\author{
Madhavi N. Pahalagedara ${ }^{\dagger}$, Lakshitha R. Pahalagedara $^{\dagger}$, Junkai He ${ }^{\ddagger}$, Ran Miao ${ }^{\dagger}$, \\ Becca Gottlieb ${ }^{\dagger}$, Dinithi Rathnayake ${ }^{\dagger}$, and Steven L. Suib ${ }^{\dagger, \neq, *}$ \\ 'Department of Chemistry, University of Connecticut, U-3060, 55 North Eagleville Rd., Storrs, \\ Connecticut 06269 (USA), Fax: (+1) (860)-486-2981, E-mail: steven.suib@uconn.edu. \\ ${ }^{\ddagger}$ Institute of Materials Science, University of Connecticut, U-3060, 55 North Eagleville Rd., \\ Storrs, Connecticut 06269 (USA)
}


KEYWORDS: Nanocomposite, Azoxybenzene, Selective reduction, Magnetically recyclable, Turnover number

\section{INTRODUCTION}

Selective catalytic reduction of nitrobenzene is an important reaction for the synthesis of intermediates and is a precursor to various industrially valuable products such as aniline, nitroso benzene, azobenzene, and azoxybenzene. ${ }^{1}$ Among them, azoxybenzene is one of the most important and essential building blocks of naturally occurring compounds and functional materials due to its conjugated system and polar functionality. ${ }^{2-4}$ In addition, azoxybenzene is valuable both as an intermediate and high-value compound widely used in industry such as dyes, reducing agents, polymerization inhibitors, and chemical stabilizers. Some azoxybenzene derivatives are used as liquid crystals in electronic displays and therapeutic medicines. ${ }^{5,6}$ Moreover, these types of compounds are the precursors for Wallach rearrangements, which offer a simple route for synthesizing hydroxyazobenzene. ${ }^{7,8}$

Azoxybenzene is produced as an intermediate in the oxidation of aniline or the reduction of nitroarenes via the condensation of nitrosobenzene and hydroxylamine. These reaction steps are quite complex and lead to different intermediates. Therefore, manipulation of reaction conditions while controlling the selectivity to the azoxy product in the midst of all possible reduction/oxidation products becomes an important research interest. ${ }^{9}$ 
Various methods have been reported for oxidative coupling of aniline using different oxidants like peraacetic acid, ${ }^{10} \mathrm{~Pb}(\mathrm{OAc}) 4{ }_{4}{ }^{11}$ and different types of molecular sieves. ${ }^{12,13}$ Reducing nitroarenes by alkaline metal borohydrides and metals such as samarium and thallium were also reported in a few studies. ${ }^{14}$ However, most of these methods lack environmental benignity and recyclability of the catalyst. Therefore, finding alternative, more benign catalysts that work at atmospheric pressure and comparatively low temperature for producing azoxybenzene with high conversion and selectivity are in high demand.

Herein, we report the preparation of an urchin-like $\mathrm{Ni} / \mathrm{Graphene}$ nanocomposite for the selective reduction of nitrobenzene to azoxybenzene with a turnover number of 36.2. The nanosize of these materials leads to a high surface area to volume ratio and therefore, to an enhanced contact between reactants and catalyst, which increases the activity dramatically. In this study, hydrazine hydrate $\left(\mathrm{N}_{2} \mathrm{H}_{4} \cdot \mathrm{H}_{2} \mathrm{O}\right)$ is used which can be considered as a very suitable reagent for the reduction of nitro groups, since only $\mathrm{N}_{2}$ and water are generated as by-products. ${ }^{15}$ Graphene acts as a promising catalyst support with a high surface area on which the metal nanocatalyst can be stabilized. Graphene also prevents metal nanoparticle aggregation and provides a desirable chemical interface between the catalyst and the reaction media thus results in enhanced catalytic performance. Moreover, the catalyst is magnetic and could be easily retrieved from the reaction mixture and reused by simple magnetic separation after the completion of the reaction.

\section{EXPERIMENTAL SECTION}

\subsection{Synthesis of Graphene oxides (GO).}


GO was synthesized from natural graphite using a modified Hummers method. Briefly, graphite powder $(1.0 \mathrm{~g})$ and $\mathrm{NaNO}_{3}(2.0 \mathrm{~g})$ were mixed and added into concentrated $\mathrm{H}_{2} \mathrm{SO}_{4}(48 \mathrm{~mL} ; 98 \%)$ in an ice bath. Under vigorous stirring, $\mathrm{KMnO}_{4}(4.0 \mathrm{~g})$ was gradually added and the temperature of the mixture was kept below $20^{\circ} \mathrm{C}$. After removing the ice bath, the mixture was stirred at $35^{\circ} \mathrm{C}$ in a water bath for $6 \mathrm{~h}$. Then, $200 \mathrm{~mL}$ water was slowly added to the mixture in an ice bath. After the dilution, $15 \mathrm{~mL}$ of $30 \% \mathrm{H}_{2} \mathrm{O}_{2}$ was added to the mixture and stirred for another $2 \mathrm{~h}$. The mixture was filtered and washed with distilled water and then re-dispersed in water by ultrasonication. Finally the product was separated by centrifugation and vacuum dried at $45^{\circ} \mathrm{C}$ for $48 \mathrm{~h}$.

\subsection{Synthesis of urchin-like Ni nanomaterial.}

First, $\mathrm{NiCl}_{2} \cdot 6 \mathrm{H}_{2} \mathrm{O}$ was dispersed in $10 \mathrm{~mL}$ of ethanol in order to prepare a $0.25 \mathrm{M}$ solution. Then, $1 \mathrm{~mL}$ of $\mathrm{KOH}$ solution $(0.2 \mathrm{M})$ and $1 \mathrm{~mL}$ of hydrazine hydrate solution $(85 \%)$ was added under vigorous stirring at $60^{\circ} \mathrm{C}$ and the obtained mixture was refluxed at $100^{\circ} \mathrm{C}$ for $2 \mathrm{~h}$. The reaction was cooled to room temperature, and the resultant product was washed with distilled water and ethanol for three times and separated by centrifugation. Finally the product was dried in a vacuum oven at $60^{\circ} \mathrm{C}$ for $12 \mathrm{~h}$.

\subsection{Synthesis of urchin-like Ni/ Graphene nanocomposite.}

About $15 \mathrm{~mL}$ of $1 \mathrm{mg} / \mathrm{mL}$ GO suspension was prepared by ultrasonication and the resultant solution was added to the $0.25 \mathrm{M} \mathrm{NiCl}_{2} \cdot 6 \mathrm{H}_{2} \mathrm{O}$ solution and the same procedure was followed to synthesize the Ni/ Graphene nanocomposite. 


\subsection{Catalyst Characterization}

Powder X-ray diffraction (XRD) analyses were performed on a Rigaku Ultima IV diffractometer with $\mathrm{Cu} \mathrm{K} \alpha(\lambda=0.15406 \mathrm{~nm})$ radiation with a beam voltage of $40 \mathrm{kV}$ and a beam current of 44 $\mathrm{mA}$. Continuous scans were taken in a $2 \theta$ range of $5-80^{\circ}$ with a scanning rate of $2.0^{\circ} \mathrm{min}$, and the phases were identified using the International Center for Diffraction Data (ICDD) database. Surface morphologies of the synthesized materials were studied using a field emission scanning electron microscope (FE-SEM) on a Zeiss DSM 982 Gemini instrument with a Schottky emitter at an accelerating voltage of $2.0 \mathrm{kV}$ with a beam current of $1.0 \mathrm{~mA}$. The samples were ultrasonically suspended in absolute ethanol and dispersed on Au-coated silicon chips and then dried overnight under vacuum. The EDX analysis was done using an Oxford X-max80 EDX analyzer operating at an electron accelerating voltage of $10 \mathrm{kV}$.

Transmission electron microscopy (TEM) was used to examine the surface morphologies and the crystalline structures of the materials using a JEOL 2010 instrument with an accelerating voltage of $200 \mathrm{kV}$. The samples were prepared by dispersing the material in ethanol. Then a drop of the dispersion was placed on a carbon coated copper grid and allowed to dry before analysis.

The nitrogen sorption experiments were performed using Quantachrome Autosorb $\mathrm{iQ}_{2}$ instrument instrument using $\mathrm{N}_{2}$ gas as the adsorbate at $77 \mathrm{~K}$ by a multipoint method. The Brunauer- Emmett-Teller (BET) method was used to determine the specific surface area and the Barrett-Joyner-Halenda $(\mathrm{BJH})$ desorption method was used to calculate the pore size distribution and pore volume. Prior to the analysis, all the samples were degassed at $150^{\circ} \mathrm{C}$ for $12 \mathrm{~h}$ in order to remove any adsorbed species.

Raman measurements were taken at room temperature on a Renishaw 2000 Ramascope attached to a charge-coupled device (CCD) camera, with an $\mathrm{Ar}^{+}$ion laser $(514.4 \mathrm{~nm})$ as the excitation source. Before each measurement was taken, the spectrometer was calibrated with a silicon 
wafer. Curve fitting for the determination of spectral parameters was performed with the software program GRAMS/32.

Thermogravimetric mass spectrometry (TG-MS) was studied using a Netzsch Libra TG209 F1 thermogravimetric analyzer coupled to a Netzsch Aëolos QMS 403C quadrupole mass spectrometer. Approximately $15 \mathrm{mg}$ of sample was loaded into an alumina crucible. Air was flowed through the sample chamber at 50 SCCM while the temperature was ramped from room temperature to $850^{\circ} \mathrm{C}$ at a rate of $10^{\circ} \mathrm{C}$ per minute while $\mathrm{TG}$ and MS signals were recorded.

\subsection{Catalytic Activity Measurements.}

Liquid phase reduction of nitrobenzene was carried out at room temperature $\left(25^{\circ} \mathrm{C}\right)$ in a double neck round bottom flask in an oil bath using $0.025 \mathrm{~g}$ catalyst, $5 \mathrm{~mL}$ ethanol, $22.5 \mathrm{mmol}$ hydrazine and $15 \mathrm{mmol}$ nitrobenzene. Small aliquots of were collected from the reaction mixture at regular intervals for analysis. For the reusability test, the spent catalyst was recovered from the reaction mixture by magnetic separation and washed thoroughly with acetone and dried at $120^{\circ} \mathrm{C}$ for $12 \mathrm{~h}$. The gas chromatography-mass spectroscopy (GC-MS) method was used for the quantitative analysis and identification of the reaction product. Analyses were performed using a HP 5971 mass selective detector coupled to a HP 5890 Series II gas chromatograph equipped with a thermal conductivity detector (TCD) through an HP-1 (nonpolar cross-linked methyl siloxane) column with dimensions of $12.5 \mathrm{~m} \times 0.2 \mathrm{~mm} \times 0.33 \mu \mathrm{m}$.

\section{RESULTS}

\subsection{Structure and characterization}


XRD patterns of as synthesized $\mathrm{Ni}$ nanomaterial and Ni/G nanocomposite are displayed in Figure 1. Both samples exhibit three well-resolved peaks at $2 \theta=44.5^{\circ}, 51.8^{\circ}$, and $76.4^{\circ}$, which coincide with the (111), (200), and (220) planes of the pure face centered cubic (fcc) structure. No other peaks were observed, indicating the high purity of as-prepared samples. An XRD pattern of commercial Ni powder is also given as a reference (Figure 1a). Ni/G nanocomposite sample shows an additional small and low broad peak around $2 \theta=26.5^{\circ}$, which can be indexed into the disordered amorphous graphene sheets. This suggests that graphene is successfully incorporated into the Ni nanomaterial. ${ }^{16}$

As synthesized $\mathrm{Ni}$ nanomaterial, $\mathrm{Ni} / \mathrm{G}$ nanocomposite was analyzed by $\mathrm{N}_{2}$ sorption measurements to study their textural properties and pore size distributions. $\mathrm{N}_{2}$ adsorption/desorption isotherms and $\mathrm{BJH}$ pore size distributions of the obtained materials are presented in Figures $2 \mathrm{a}$ and $\mathrm{b}$. The BET surface areas of $\mathrm{Ni}$ nanomaterial and Ni/G nanocomposite are 16 and $93 \mathrm{~m}^{2} / \mathrm{g}$, respectively. Upon incorporation of graphene, pore volume also has been increased from 0.08 to $0.17 \mathrm{~cm}^{3} / \mathrm{g}$ (Table 1). Ni/G nanocomposite shows a larger $\mathrm{N}_{2}$ uptake than the $\mathrm{Ni}$ sample and indicates a higher mesopore volume. The Ni/G nanocomposite resembles a Type-H3 IUPAC classification, which may result from slit-shaped pores between parallel layers in graphene. ${ }^{17,18}$ The BJH pore diameter showed a slight increase upon graphene incorporation. However, Ni/G nanocomposite possesses a higher amount of pores in the mesoporous region, with a uniform size distribution (Figures 2a). $\mathrm{N}_{2}$ adsorption/desorption isotherms and $\mathrm{BJH}$ pore size distribution curves of as synthesized GO sample are given in Figure S1.

As synthesized samples were examined by Raman spectroscopy and results are given in Figure 3. Ni nanomaterial did not show any detectable peaks in the region between $800-2000 \mathrm{~cm}^{-1}$. 
Raman spectra of GO and Ni/G nanocomposite give 2 intense peaks at 1350 and $1600 \mathrm{~cm}^{-1}$ (Figure $3 \mathrm{~b}$ and $\mathrm{c}$ ). The intensity ratio between the bands at 1350 and $1600 \mathrm{~cm}^{-1}$ has been decreased upon reduction with hydrazine.

The surface morphologies of as prepared $\mathrm{Ni}$ nanomaterial and $\mathrm{Ni} / \mathrm{G}$ nanocomposite were examined by FE-SEM and TEM. According to Figure $4 a$ and $b$, the Ni nanomaterial shows a rough surface with an urchin-like morphology. Figure 4c gives the TEM image of the Ni/G nanocomposite. A typical flake-like morphology of graphene sheets and urchin-like Ni nanomaterial are uniformly dispersed on the graphene surface. Figure $4 \mathrm{~d}$ confirms the polycrystalline nature of the $\mathrm{Ni} / \mathrm{G}$ nanocomposites in the selected area. The sharp rings in the SAED pattern can be indexed as (111), (200), (220), and (311) reflections with plane distances of $0.20,0.17,0.12$ and $0.10 \mathrm{~nm}$, respectively. This indicates d-spacings for pure cubic Nickel and matches the d-spacing values obtained from XRD studies.

The thermogravimetric mass spectrometry (TG-MS) analysis profiles of Ni nanomaterials and $\mathrm{Ni} / \mathrm{G}$ nanocomposites are given in Figure 5. When these materials are heated under air, a small weight loss was observed below $200^{\circ} \mathrm{C}$. Both samples begin to show weight gain at about $400^{\circ} \mathrm{C}$. According to TG-MS data, the weight gain difference between Ni nanomaterials and Ni/G nanocomposites is about 2.48\%. In its $\mathrm{CO}_{2}$ evolution profile, Ni nanomaterial shows a single peak between $200-300^{\circ} \mathrm{C}$. On the other hand, $\mathrm{Ni} / \mathrm{G}$ nanocomposite shows two peaks, between 200- $300^{\circ} \mathrm{C}$ and between $350-550^{\circ} \mathrm{C}$ with a markedly high intensity.

\subsection{Catalytic activity}


The catalytic activity of $\mathrm{Ni}$ and $\mathrm{Ni} / \mathrm{G}$ nanocomposite towards the reductive coupling of nitrobenzene to azoxybenzene was investigated at room temperature and at atmospheric pressure using ethanol as the solvent and hydrazine monohydrate as the reducing agent. Activities of as synthesized Ni nanomaterials and Ni/G nanocomposites in nitrobenzene reduction were tested and the obtained results are summarized in Table $2 . \mathrm{Ni} / \mathrm{G}$ nanocomposite gave $100 \%$ conversion with $100 \%$ selectivity towards the desired azoxy product in $2 \mathrm{~h}$; therefore all the reactions were carried out for two hours under the same experimental conditions. Ni nanomaterial gave a $73 \%$ nitrobenzene conversion with $80 \%$ selectivity towards azoxybenzene (Entry 1). Commercial Ni could only give the azoxy product with a conversion of $2 \%$ (18\% selectivity) while aniline was the major product.

A series of catalytic experiments was carried out using $\mathrm{Ni} / \mathrm{G}$ nanocomposites in order to understand the variation of nitrobenzene to azoxybenzene as a function of time, temperature and nitrobenzene: hydrazine molar ratio. Figure 6 gives the time-conversion plot for nitrobenzene reduction at room temperature. Here, aniline and azobenzene were formed as by-products; however they were gradually converted to azoxybenzene with increasing time. The same trend was observed with $\mathrm{Ni}$ nanomaterial (Figure 6a), but nitrobenzene conversion rate was low compared to the previous catalyst. After $24 \mathrm{~h}$ of reaction, Ni nanomaterial catalyst exhibited $100 \%$ conversion with $88 \%$ azoxybenzene selectivity (Table 2, entry 2). Although the reactions performed at different temperatures $\left(50^{\circ} \mathrm{C}\right.$ and $\left.80^{\circ} \mathrm{C}\right)$, gave $100 \%$ conversions, the selectivities drop due to the formation of azobenzene as the major side product (Figure S2a). Then, the effect of nitrobenzene to hydrazine molar ratio was studied at room temperature while keeping all the other experimental parameters constant. When the nitrobenzene : hydrazine molar ratio was adjusted to $1: 0.5$, the conversion was comparatively low (75\%) with the azoxybenzene 
selectivity of $85 \%$ and aniline selectivity of $15 \%$. Conversion (100\%) and selectivity towards the desired azoxybenzene product was observed when the ratio was 1:1.5. With a further increase up to $1: 2.5$, although the conversion was $100 \%$, azoxybenzene selectivity decreased significantly to $70 \%$, due to the formation of aniline as the major side product (Figure S2b).

In order to calculate the rate constant for the catalytic reduction of nitrobenzene, $\ln \left(\mathrm{C}_{\mathrm{t}} / \mathrm{C}_{0}\right)$ was plotted against time for the $\mathrm{Ni}$ nanomaterial and the Ni/G nanocomposite (Figure S3). $\mathrm{C}_{0}$ is the initial concentration of nitrobenzene and $C_{t}$ is the concentration of nitrobenzene at time t. The linear fit with a coefficient of determination very close to unity supports the pseudo-first-order kinetics. The reaction rate constants $k$, calculated using the rate equation $\ln \left(\mathrm{C}_{t} / \mathrm{C}_{0}\right)=k t$, are $1.2 \times$ $10^{-2}$ and $4.5 \times 10^{-2} \mathrm{~min}^{-1}$ for $\mathrm{Ni}$ nanomaterial and $\mathrm{Ni} / \mathrm{G}$ nanocomposite catalysts, respectively. Incorporation of graphene has increased the reaction rate for the reduction of nitrobenzene to azoxybenzene, nearly 4 fold compared to the catalyst without graphene. When increasing the nitrobenzene: hydrazine molar ratio from 1:1.5 to 1:2.5, the conversion was not changed suggesting that the reaction is zeroth order with respect to the reductant. Blank experiments were performed with graphene and in the absence of the catalyst in order to confirm the catalytic nature of the present catalyst. Graphene showed a 5\% conversion after $2 \mathrm{~h}$. Reaction carried out without the catalyst gave a very small conversion (1\%). In both reactions, aniline was the only product detected from GC (Table 2, entry $10 \& 11)$.

To illustrate the general applicability of the Ni/G nanocomposite, the scope of the reaction was extended to reductive coupling of a range of nitroarenes (Table 3). Under the optimized conditions, a variety of structurally different nitroarenes was selectively transformed to their corresponding azoxy products with a high conversion and selectivity regardless of the presence of electron donor or acceptor groups in the phenyl ring. 
The reusability was tested by recycling the spent catalyst in consecutive runs. The catalyst was recovered from the reaction mixture by magnetic separation (Scheme 1) and washed thoroughly with acetone and dried at $120^{\circ} \mathrm{C}$ for $12 \mathrm{~h}$. Over a period of four reaction cycles under the same conditions, no significant loss of activity was observed (93\% conversion, $100 \%$ selectivity after the $4^{\text {th }}$ reuse). XRD patterns of the catalyst before and after the reaction are given in Figure S4 and the catalyst did not show any obvious change.

\section{DISCUSSION}

This study reports the synthesis of magnetic Ni/G nanocomposites that act as a promising catalyst in the selective reduction of nitrobenzene to azoxybenzene at room temperature and under atmospheric pressure. Here, the composite is synthesized via the reduction of $\mathrm{Ni}^{2+}$ and GO in a single step where hydrazine monohydrate is used as the reducing agent. ${ }^{19,20}$ Oxygen containing groups in $\mathrm{GO}$, such as $-\mathrm{OH}$ and $-\mathrm{COOH}$ facilitate the homogeneous dispersion of $\mathrm{Ni}^{2+}$ on its surface and both $\mathrm{Ni}^{2+}$ and GO are simultaneously reduced by hydrazine. Therefore, this can be considered as an in situ reduction growth process. Raman spectroscopy is widely used to characterize crystal structure, disorder and defects in graphene-based materials. For example, the reduction process of GO can manifest itself in Raman spectra by the changes in relative intensity of two main peaks: D and G. We use this information to verify the reduction process. Fig. 3 shows the Raman spectra of $\mathrm{GO}$ and Ni/G nanocomposite which contains reduced GO. Both spectra show the existence of the $\mathrm{G}$ band, related to the $\mathrm{C}-\mathrm{C}$ vibrations of carbon with a $\mathrm{sp}^{2}$ orbital structure, and the D band (disorder band), related to the disorder-induced vibration of the $\mathrm{C}-\mathrm{C}$ bond. The decrease in the intensity ratio between $\mathrm{D}$ band $\left(1350 \mathrm{~cm}^{-1}\right)$ and $\mathrm{G}$ band $\left(1600 \mathrm{~cm}^{-}\right.$

${ }^{1}$ ) in Raman spectroscopy confirms the successful reduction of GO by hydrazine and is in 
agreement with previous reports. ${ }^{21,22}$ Due to its remarkable physical properties, graphene has been used as an ideal substrate to anchor inorganic nanoparticles for a wide range of applications..$^{23-27}$

According to SEM images, the in situ reduction growth process has produced Ni nanomaterials with an urchin-like surface morphology. TEM studies further confirm that incorporation of graphene does not radically change their shape or surface morphology. Enhancement of the catalytic activity by graphene incorporation can be explained in two ways. As a catalyst support, there is inhibition of the aggregation of active Ni nanoclusters by dispersing them on the surface and providing a desirable chemical interface between the catalyst and the reaction media. On the other hand, graphene can adsorb nitrobenzene via $\pi-\pi$ stacking interactions, and again improves contact between the reactant and the catalyst surface and facilitates transport of electrons from graphene to the Ni nanomaterial. ${ }^{28}$ These factors lead to the accumulation of a high concentration of nitroaromatic compounds over the active Ni surface, being subject to efficient reduction to the corresponding azoxy compound. ${ }^{29-31}$ The higher activity of Ni/G nanocomposite can be attributed to its accessible surfaces toward the substrate molecules due to their greater surface/ volume ratio compared to that of Ni nanomaterial. Therefore, the enhanced catalytic activity of the urchin-like $\mathrm{Ni} / \mathrm{G}$ composite may be associated with the uniform and organized microstructure.

Small weight losses observed below $200^{\circ} \mathrm{C}$ in TG-MS profiles are due to the removal of physisorbed water and other gaseous species. Both Ni nanomaterials and Ni/G nanocomposites show weight gains after $400^{\circ} \mathrm{C}$ and this can be attributed to the oxidation of $\mathrm{Ni}$ material to $\mathrm{NiO}$. Compared to the typical initial oxidation temperature of bulk nickel materials $\left(600^{\circ} \mathrm{C}\right)$, the oxidation of the nanomaterial occurs at much lower temperature. According to TG-MS data, the weight gain difference between $\mathrm{Ni}$ nanomaterial and $\mathrm{Ni} / \mathrm{G}$ nanocomposite is about $2.48 \%$. This 
number agrees well with the initial weight ratio of $\mathrm{G} / \mathrm{Ni}$, which is about $2.52 \%$. In their $\mathrm{CO}_{2}$ evolution profile, both $\mathrm{Ni}$ nanomaterial and Ni/G nanocomposite show a peak between 200$300^{\circ} \mathrm{C}$. This can be attributed to the oxidation of amorphous carbon obtained due to the reduction of solvent. In contrast to that, only the $\mathrm{Ni} / \mathrm{G}$ nanocomposite shows a peak between $350-550^{\circ} \mathrm{C}$, with a markedly high intensity due to the oxidation of graphene to $\mathrm{CO}_{2}{ }^{32,33}$

$\mathrm{Ni} / \mathrm{G}$ nanocomposite showed the highest catalytic activity for the synthesis of azoxybenzene with $100 \%$ selectivity. Although it takes $2 \mathrm{~h}$ to give a complete conversion, about $65 \%$ of nitrobenzene is converted within the first 15 minutes. According to the obtained results, azoxybenzene could be found in the reaction mixture even at initial stages and uggests that there is no induction period for this catalyst. Aniline and azobenzene were found as side products; however they were gradually transformed to azoxybenzene with time. The reason for this enhanced activity is the presence of graphene. Graphene can interact with nitrobenzene via $\pi-\pi$ stacking interactions, and improve contact between the reactant and the catalyst surface which increase the reaction rate. Moreover, the formed aromatic intermediates (nitrosobenzene and phenylhydroxylamine) can be easily coupled on the graphene surface. Even though a higher concencentartion of hydrazine is used to reduce the graphene oxide to graphene in the initial synthesis step, a small amount of GO can be remaining. This remaining GO can act as an oxidizing agent to oxidize aniline and azobenzene which were produced as side products. This makes the Ni/G nanocomposite $100 \%$ selective towards azoxybenzene formation.

The mechanism of the catalyzed reduction of nitroarenes to azoxybenzene can be explained by a redox mechanism (Scheme S1). As a reagent, hydrazine is oxidized generating electrons necessary for the reduction and $\mathrm{N}_{2}$ gas and water are produced. These electrons reduce nitrobenzene (1) to nitrosobenzene (2) and further reduction results in the formation of 
phenylhydroxylamine (3). Condensation of (2) and (3) forms azoxybenzene (4) as the major product in the reaction medium. Further reduction of (3) and (4) may produce aniline (5) and azobenzene (6) as side products. According to catalytic data (Table 2), these side products are formed and promoted by higher temperatures and higher concentrations of hydrazine. This reaction is carried out at room temperature and with a controlled amount of the reducing agent which disfavor over reduction. Therefore, this can be considered as a promising approach to obtain the azoxy product with a high selectivity (100\%) in the midst of all possible reduction products.

Recyclability studies prove that the Ni/G nanocomposite stays intact even after multiple repeated cycles with no leaching effects. Another remarkable feature of the Ni/G nanocomposite is that this material can be readily isolated from the reaction medium due to its magnetic nature. Catalyst reusability is of major importance in heterogeneous catalysis and development of new technologies for catalyst separation and recycling to substitute traditional time- and solventconsuming procedures is a challenge for green chemistry. Therefore, the catalyst described here can be considered as an environmentally friendly green catalyst which provides a convenient route for the catalyst recovery by the application of an external permanent magnet. ${ }^{34,35}$

\section{CONCLUSIONS}

In conclusion, an efficient catalytic process to selectively synthesize azoxybenzene under mild reaction conditions has been reported. Ni/G nanocomposite material exhibited the highest catalytic performance giving $100 \%$ conversion with $100 \%$ selectivity towards the desired product in $2 \mathrm{~h}$ at room temperature and under atmospheric pressure. This catalyst system can be 
applied to reduce diverse types of substituted nitroarenes into their corresponding azoxy products. Ni/G nanocomposite is magnetically separable and can be reused several times without any significant loss of its initial catalytic potential. Therefore, this catalytic system offers several advantages for the preparation of azoxybenzenes from the corresponding nitroarene such as mild conditions, low catalyst loading, high conversions and selectivities, which yield an efficient and attractive methodology for organic synthesis.

\section{Appendix A. Supplementary data}

$\mathrm{N}_{2}$ adsorption/desorption isotherms and $\mathrm{BJH}$ pore size distribution curves of as synthesized GO sample; Effect of temperature and nitrobenzene: hydrazine molar ratio on nitrobenzene reduction; Plots of $\ln \left(\mathrm{C}_{\mathrm{t}} / \mathrm{C}_{0}\right)$ versus time for the catalytic reduction of nitrobenzene at room temperature with $\mathrm{Ni}$ nanomaterial and $\mathrm{Ni} / \mathrm{G}$ nanocomposite; XRD patterns of the fresh $\mathrm{Ni} / \mathrm{G}$ nanocomposite catalyst and the recovered catalyst; Reaction scheme for the reductive coupling of nitrobenzene.

\section{ACKNOWLEDGMENTS}

We thank the Geosciences and Biosciences Division, Office of the Basic Energy Sciences, Office of Science, and U.S. Department of Energy for supporting this work under grant DEFG02-86ER13622-A000. 


\section{REFERENCES}

1. A. Grirrane, A. Corma, H. García, Science 322 (2008) 1661.

2. C.-F. Chang, S.-T. Liu, J. Mol. Catal. A: Chemical 299 (2009) 121.

3. F. Hamon, F. Djedaini-Pilard, F. Barbot, C. Len, Tetrahedron 65 (2009) 10105.

4. E.R. Burkhardt, K. Ma, Chem. Rev. 106 (2006) 2617.

5. S. Sakaue, T. Tsubakino, Y. Nishiyam, Y. Ishii, J. Org. Chem. 58 (1993) 3633.

6. S. Acharyya, S. Ghosh, R. Bal, ACS Sustainable Chem. Eng. 2 (2014) 584.

7. E. Buncel, Can. J. Chem. 78 (2000) 1251.

8. E. Buncel, Acc. Chem. Res, 8 (1975) 132.

9. H. Zhu, Y. Lu, F. Fan, S. Yu, Nanoscale 5 (2013) 7219.

10. S. Ghosh, S. S. Acharyya, T. Sasaki, R. Bal, Green Chem. 17 (2015) 1867.

11. H.E. Baumgar, A. Stalk, E.M. Miller, J. Org. Chem. 30 (1965) 1203.

12. S. B. Waghmode, S. M. Sabne, S. Sivasanker, Green Chem. 3 (2001) 285.

13. A. Tuel, L. G. Hubert-Pfalzgraf, J.Cat. 217 (2003) 343.

14. Y. Liu, B. Liu, A. Guo, Z. Dong, S. Jin, Y. Lu, Synthetic Commun. 42 (2012) 2201. 
15. D. Cantillo, M.M. Moghaddam, C.O. Kappe, J. Org. Chem. 78 (2013) 4530.

16. B. Zhao, P. Liu, H. Zhuang, Z. Jiao, T. Fang, W. Xu, B. Lu, Y. Jiang, J. Mater. Chem. A 1 (2013) 367.

17. S-M. Paek, E. Yoo, I. Honma, Nano Lett. 9 (2009) 72.

18. B. Zhao, G. Zhang, J. Song, Y. Jiang, H. Zhuang, P. Liu, T. Fang, Electrochimica Acta 56 (2011) 7340 .

19. S-M. Paek, E. Yoo, I. Honma, Nano Lett. 9 (2008) 4071.

20. Y.J. Mai, J.P. Tu, C.D. Gu, X.L. Wang, J. Power Sources 209 (2012) 1.

21. J. Zhu, T. Zhu, X. Zhou, Y. Zhang, X.W. Lou, X. Chen, H. Zhang, H.H. Hng, Q. Yan, Nanoscale 3 (2011) 1084.

22. S. Stankovich, D.A. Dikin, R.D. Piner, K.A. Kohlhaas, A. Kleinhammes, Y. Jia, Y. Wu, S.T. Nguyen, R.S. Ruoff, Carbon 45 (2007) 1558.

23. W. Fan, W. Gao, C. Zhang, W.W. Tjiu, J. Pan, T. Liu, J. Mater. Chem. 22 (2012) 25108.

24. T. Xu, L. Zhang, H. Cheng, Y. Zhu, Appl. Catal. B 101 (2011) 382.

25. W. Li, Y. Gao, W. Chen, P. Tang, W. Li, Z. Shi, D. Su, J. Wang, D. Ma, ACS Catal. 4 (2014) 1261.

26.Y. Shiraishi, H. Sakamoto, K. Fujiwara, S. Ichikawa, T. Hirai, ACS Catal. 4 (2014) 2418. 
27. L. Shao, X. Huang, D. Teschner, W. Zhang, ACS Catal. 4 (2014) 2369.

28. Z. Zhang, F. Xiao, J. Xi, T. Sun, S. Xiao, H. Wang, S. Wang, Y. Liu, Scientific Reports 4 (2014) 4053.

29. Y. Zhang, S. Liu, W. Lu, L. Wang, J. Tian, X. Sun, Catal. Sci. Technol. 1 (2011) 1142.

30. J. Li, C. Liu, Y. Liu, J. Mater. Chem. 22 (2012) 8426.

31. M. Yang, X. Pan, N. Zhang, Y. Xu, Cryst. Eng. Comm. 15 (2013) 6819.

32. P. Song, D. Wen, Z.X. Guo, T. Korakianitis, Phys. Chem. Chem. Phys. 10 (2008) 5057.

33. L. Zhang, J. Zhao, M. Li, H. Ni, J. Zhang, X. Feng, Y. Ma, Q. Fan, X. Wang, Z. Hu, W. Huang, New J. Chem. 36 (2012) 1108.

34. L. Menini, M.C. Pereira, A.C. Ferreira, J.D. Fabris, E.V. Gusevskaya, Applied Catalysis A: General 392 (2011) 151.

35. M.J. Jacinto, O.H.C.F. Santos, R.F. Jardim, R. Landers, L.M. Rossi, Applied Catalysis A: General 360 (2009) 177. 
Table 1. Structural parameters of Ni nanomaterial, Ni/G nanocomposite and GO sample.

\begin{tabular}{|c|c|c|c|}
\hline Catalyst & BET SA $\left(\mathrm{m}^{2} / \mathrm{g}\right)$ & $\begin{array}{l}\text { BJH desorption pore } \\
\text { volume }\left(\mathrm{cm}^{3} / \mathrm{g}\right)\end{array}$ & $\begin{array}{l}\text { BJH desorption } \\
\text { pore diameter } \\
(\mathrm{nm})\end{array}$ \\
\hline Ni nanomaterial & 16 & 0.08 & 3.4 \\
\hline Ni/G nanocomposite & 93 & 0.17 & 3.6 \\
\hline GO & 18 & 0.04 & 4.2 \\
\hline
\end{tabular}


Table 2. Reductive coupling of nitrobenzene to azoxybenzene. ${ }^{\text {a }}$

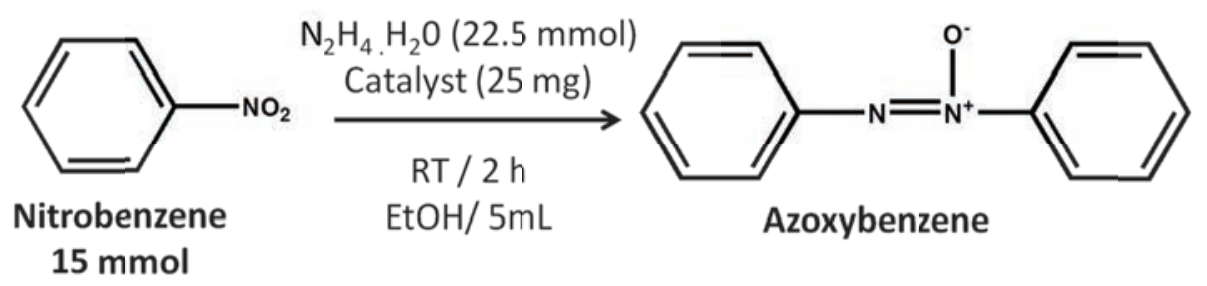

\begin{tabular}{|c|c|c|c|c|c|c|c|}
\hline \multirow[t]{2}{*}{ Entry } & \multirow[t]{2}{*}{ Catalyst } & \multirow{2}{*}{$\begin{array}{c}\text { Nitrobenzene } \\
\text { conversion } \\
(\%)^{\mathrm{h}}\end{array}$} & \multicolumn{3}{|c|}{ Selectivity $(\%)^{\mathrm{i}}$} & \multirow{2}{*}{$\begin{array}{l}\text { Turnover } \\
\text { number } \\
(\mathrm{TON})^{\mathrm{j}}\end{array}$} & \multirow{2}{*}{$\begin{array}{c}\text { Turnover } \\
\text { Frequency } \\
\text { (TOF) }^{\mathrm{k}}\end{array}$} \\
\hline & & & Aniline & $\begin{array}{c}\text { Azo } \\
\text { benzene }\end{array}$ & $\begin{array}{c}\text { Azoxy } \\
\text { benzene }\end{array}$ & & \\
\hline 1 & nanomate & 73 & 12 & 8 & 80 & 12.8 & 6.43 \\
\hline
\end{tabular}


$2 \quad$ Ni nanomaterial $^{\mathrm{b}}$

$3 \quad \mathrm{Ni} / \mathrm{G}$ nanocomposite

$4 \quad$ Commercial Ni

$5 \quad \mathrm{Ni} / \mathrm{G}$ nanocomposite ${ }^{\mathrm{c}}$

6 Ni/G nanocomposite ${ }^{d}$

$7 \quad \mathrm{Ni} / \mathrm{G}$ nanocomposite ${ }^{\mathrm{e}}$

$8 \quad$ Ni/G nanocomposite ${ }^{f}$

$9 \quad \mathrm{Ni} / \mathrm{G}$ nanocomposite ${ }^{\mathrm{g}}$

10

11
100

100

2

100

100

75

100

93

5

Graphene

No catalyst
12

0

88

17.6

100

36.2

18.10

0.15

82

0

18

0.30

18.10

36.2

44

56

36.2

15

0

85

27.1

30

0

70

36.2

$100 \quad 33.6$

16.80

100

0

0

100

0

${ }^{\mathrm{a}}$ Reaction conditions : solvent (ethanol) $=5 \mathrm{~mL}$, substrate (nitrobenzene) $=15 \mathrm{mmol}$, nitrobenzene: hydrazine (molar ratio) $=1: 1.5,0.025 \mathrm{~g}$ catalyst, room temperature, reaction time $=2 \mathrm{~h}$. Conversion and selectivity determined and confirmed by GC-MS. ${ }^{\mathrm{b}}$ Reaction after $24 \mathrm{~h}$ (TOF was not calculated). The reaction temperatures were ${ }^{\mathrm{c}} 50,{ }^{\mathrm{d}} 80^{\circ} \mathrm{C}$ respectively. Nitrobenzene: hydrazine $\left(\right.$ molar ratio) $=1: 0.5^{\mathrm{e}}$ and 1:2.5 ${ }^{\mathrm{f}}$ respectively. ${ }^{\mathrm{g}}$ Catalyst after 4 cycles. ${ }^{\mathrm{h}}$ Nitrobenzene conversion $=[$ moles of nitrobenzene converted/ moles of nitrobenzene used $] \times 100 .{ }^{i}$ Selectivity $=$ [Total moles of the product formed/ Total moles of nitrobenzene converted]. ${ }^{\mathrm{j}}$ Turnover number $(\mathrm{TON})=[$ moles of converted substrate $] \times(\text { moles of Nickel })^{-1} \cdot{ }^{\mathrm{k}}$ Turnover frequency $(\mathrm{TOF})=$ [moles of converted substrate] $x[(\text { moles of Nickel) } x \text { (reaction time in } \mathrm{h})]^{-1} \cdot(-)=$ not calculated. 
Table 3: Catalytic activity of the Ni/G nanocomposite for the formation of azoxybenzene via selective reduction of different substituted nitroarenes. ${ }^{a}$

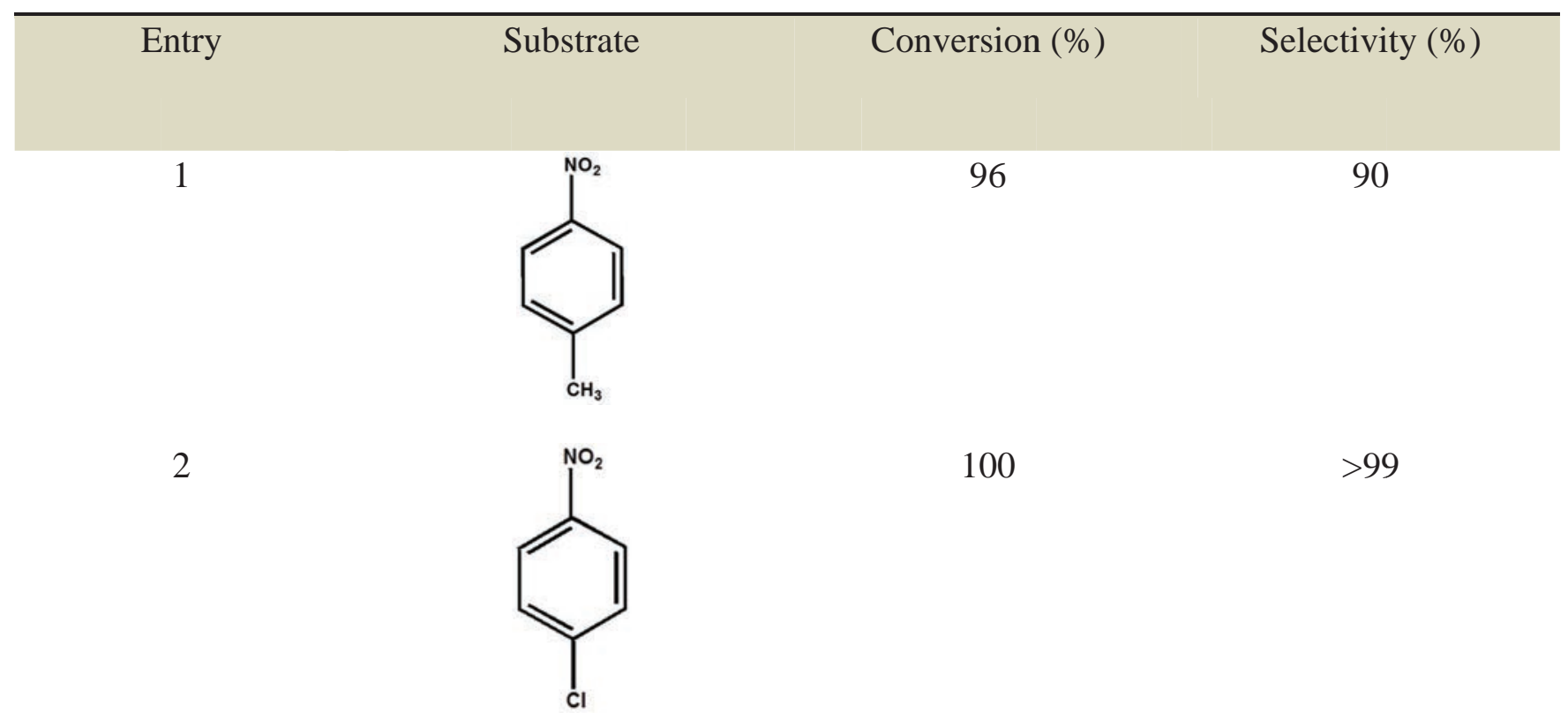


3

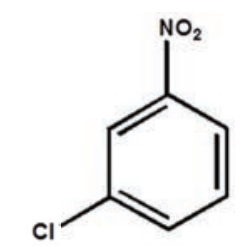

4<smiles>Nc1ccc([N+](=O)[O-])cc1</smiles>

5

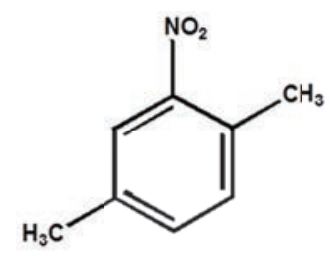

100

${ }^{\mathrm{a}}$ Reaction conditions : solvent (ethanol) $=5 \mathrm{~mL}$, substrate $=15 \mathrm{mmol}$, nitroarene: hydrazine $($ molar ratio $)=1: 1.5,0.025 \mathrm{~g}$ catalyst, room temperature, reaction time $=2 \mathrm{~h}$. Conversion and selectivity determined and confirmed by GC-MS. 


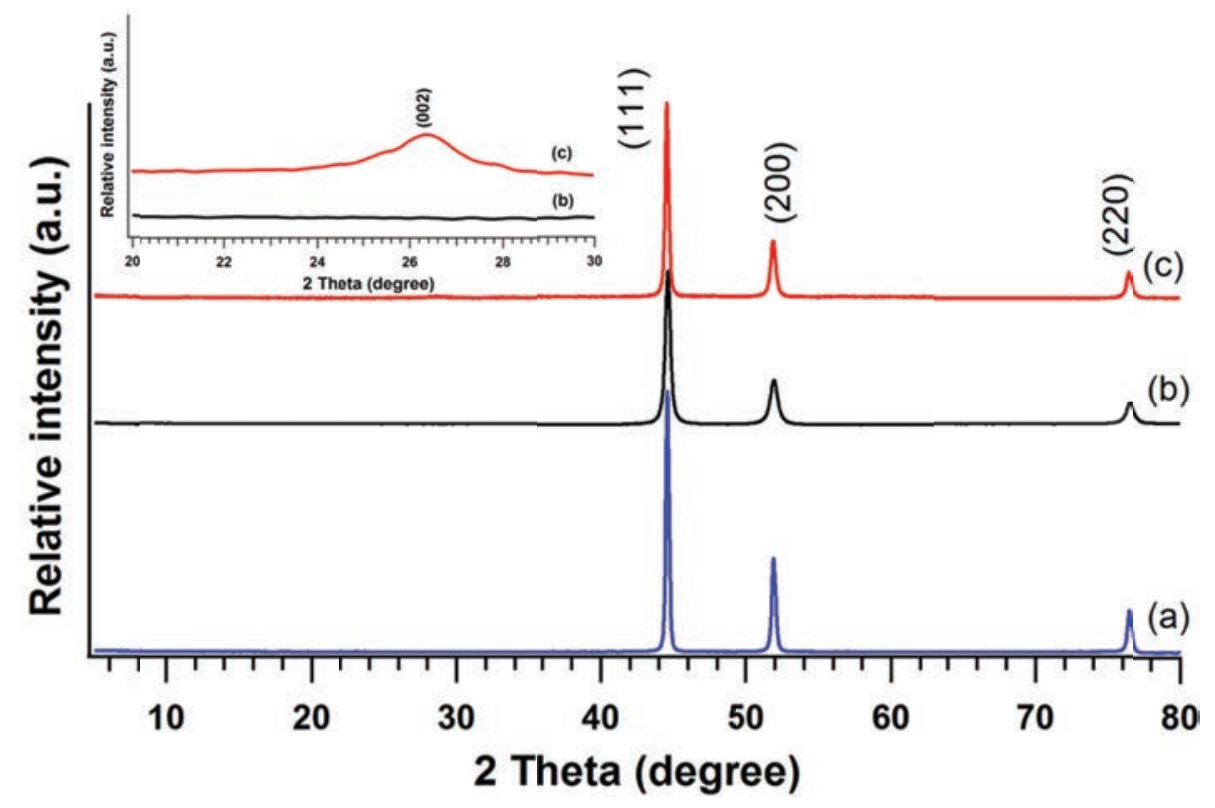

Figure 1. X-ray diffraction patterns of (a) commercial Ni powder (b) Ni nanomaterial and (c) $\mathrm{Ni} / \mathrm{G}$ nanocomposite.

(a)

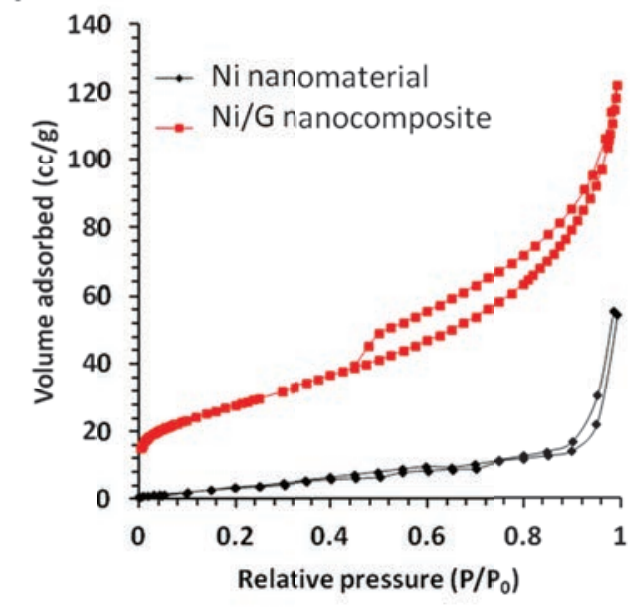

(b)

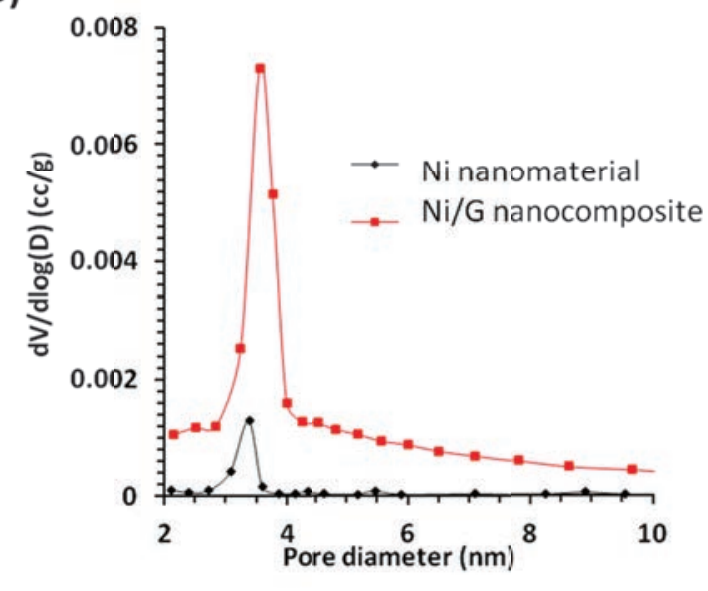

Figure 2. (a) $\mathrm{N}_{2}$ adsorption/desorption isotherms and (b) BJH pore size distribution curves of $\mathrm{Ni}$ nanomaterial and $\mathrm{Ni} / \mathrm{G}$ nanocomposite. 


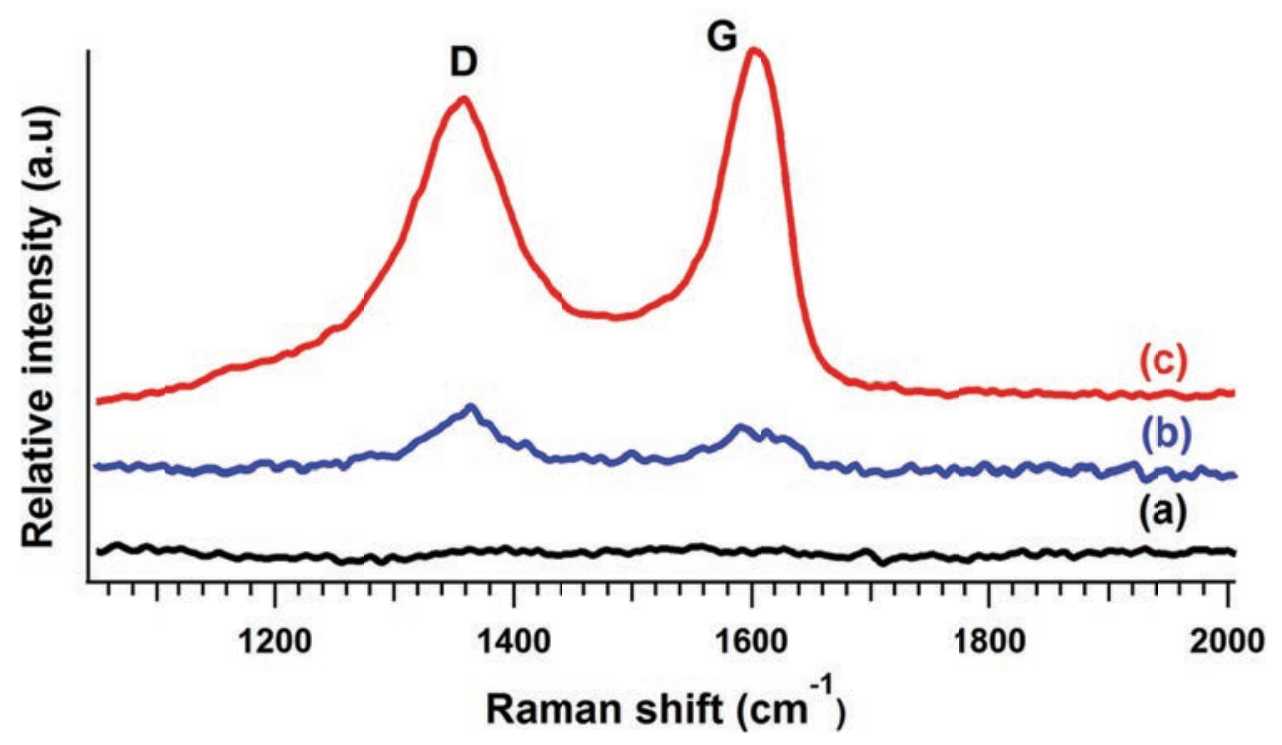

Figure 3. Raman spectra of (a) Ni nanomaterial, (b) GO and (C) Ni/G nanocomposite.
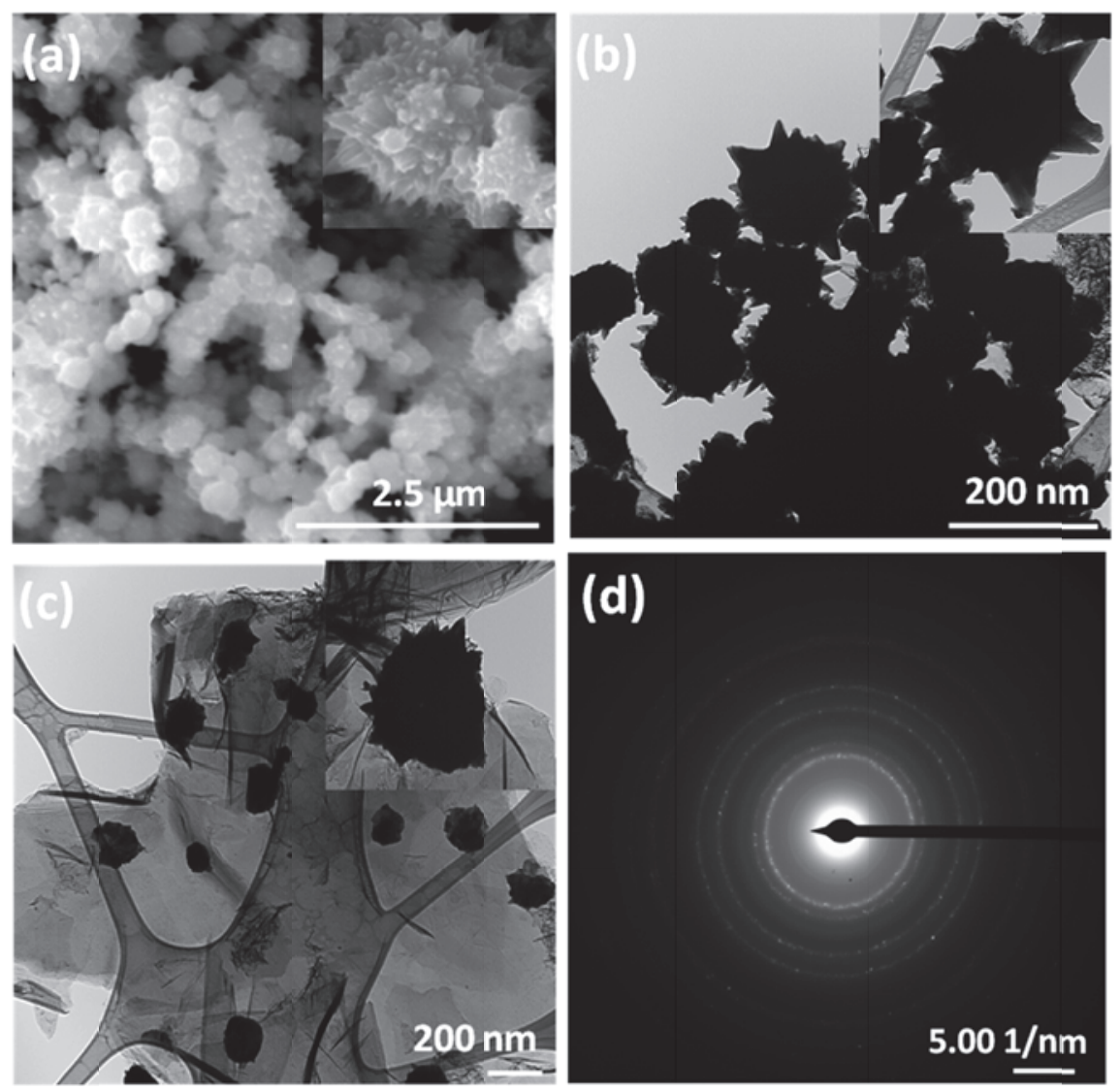

Figure 4. SEM image of (a) Ni nanomaterial, TEM images of (b) Ni nanomaterial, (c) Ni/G nanocomposite and (d) SAED pattern of Ni/G nanocomposite. 
(a)

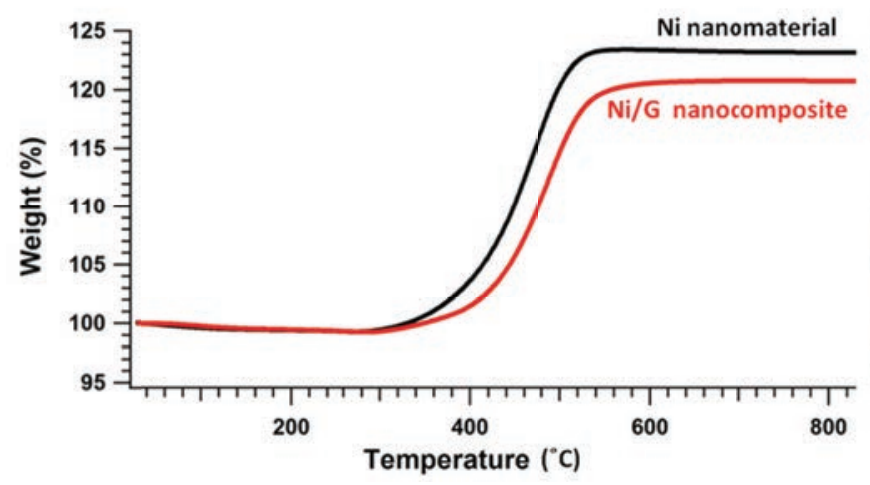

(b)

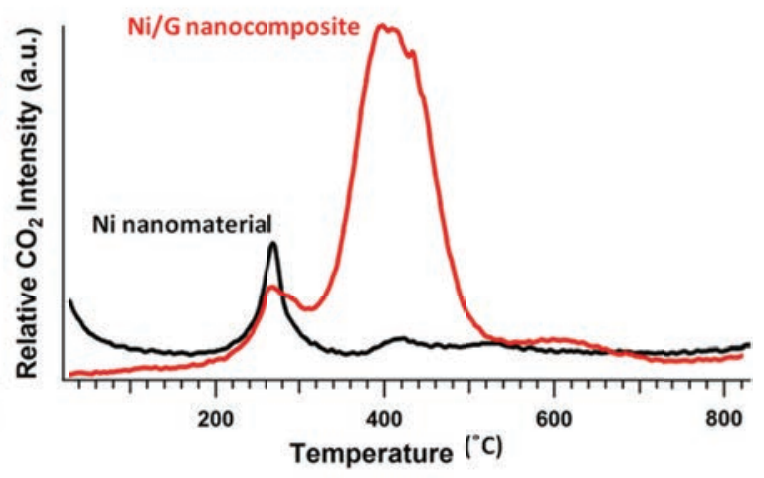

Figure 5. (a) TGA and (b) $\mathrm{CO}_{2}$ evolution profiles of $\mathrm{Ni}$ nanomaterial and $\mathrm{Ni} / \mathrm{G}$ nanocomposite.

(a)

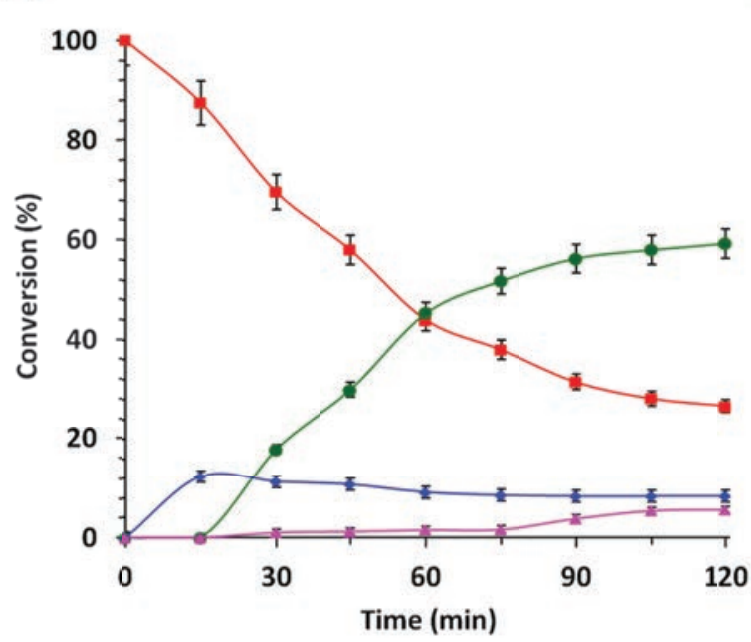

(b)

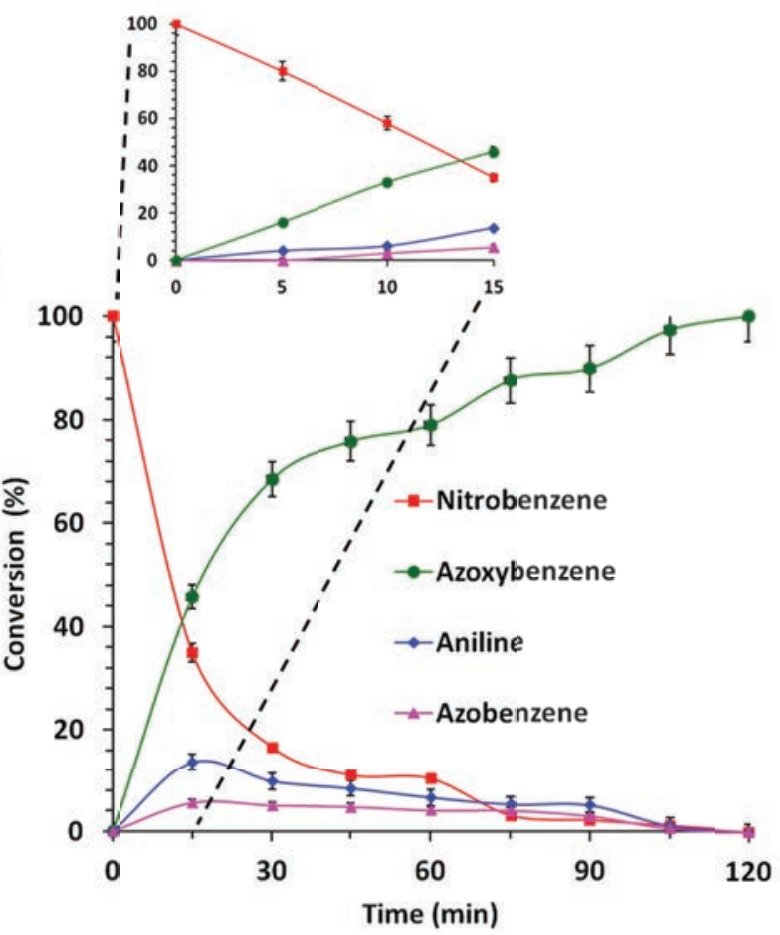

Figure 6. Time-conversion plots for nitrobenzene reduction by (a) Ni nanomaterial and (b) $\mathrm{Ni} / \mathrm{G}$ nanocomposite at room temperature. (Reaction conditions: solvent (ethanol) $=5 \mathrm{~mL}$, substrate $($ nitrobenzene $)=15 \mathrm{mmol}$, nitrobenzene: hydrazine $($ molar ratio $)=1: 1.5,0.025 \mathrm{~g}$ catalyst, room temperature, reaction time $=2 \mathrm{~h}$. Conversion and selectivity determined and confirmed by GCMS) 


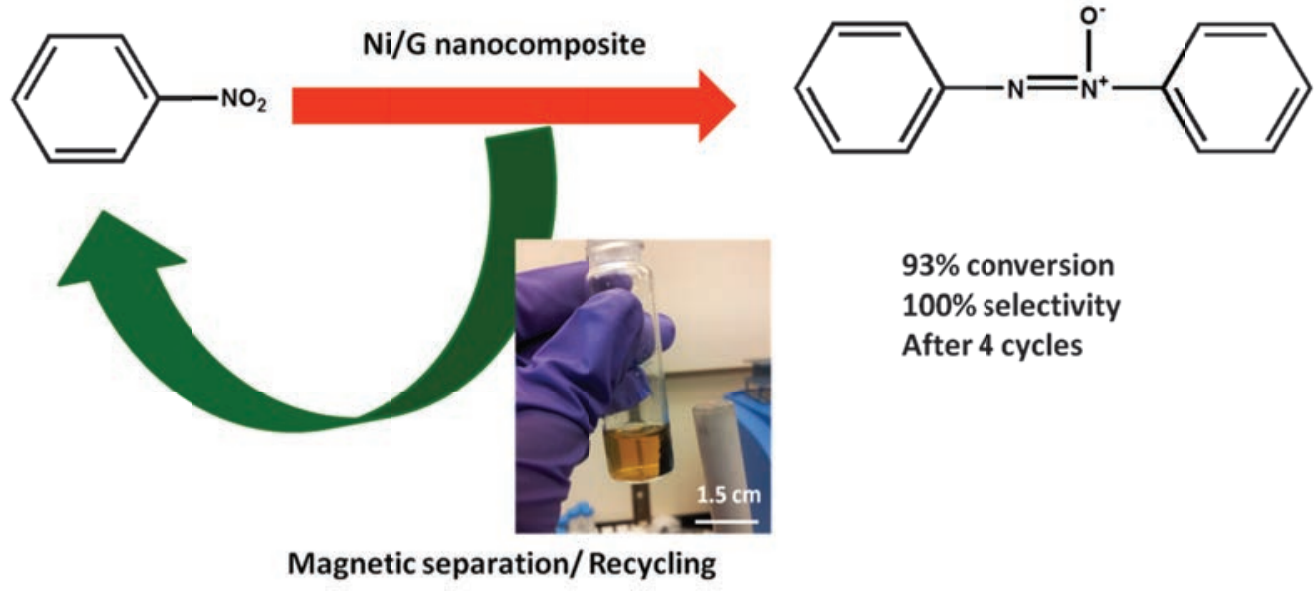

Scheme 1. Magnetic separation and recycling of the Ni/G nanocomposite. 


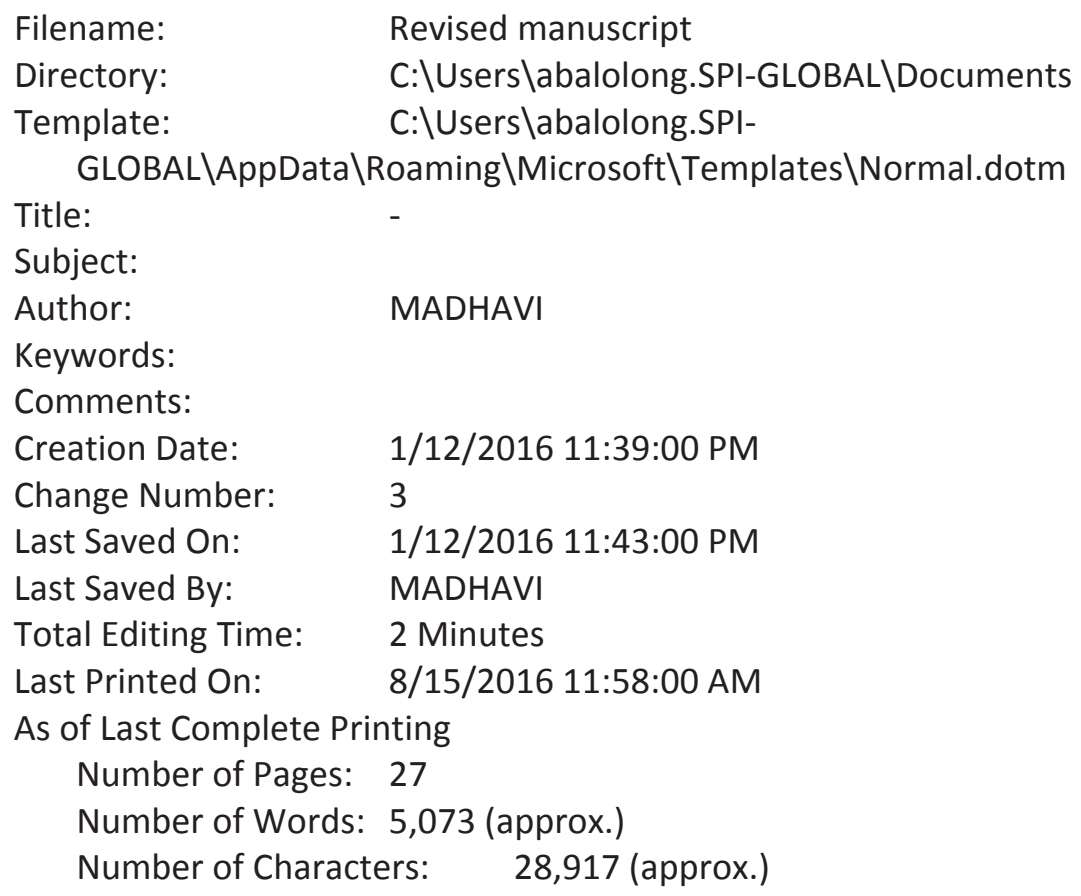

